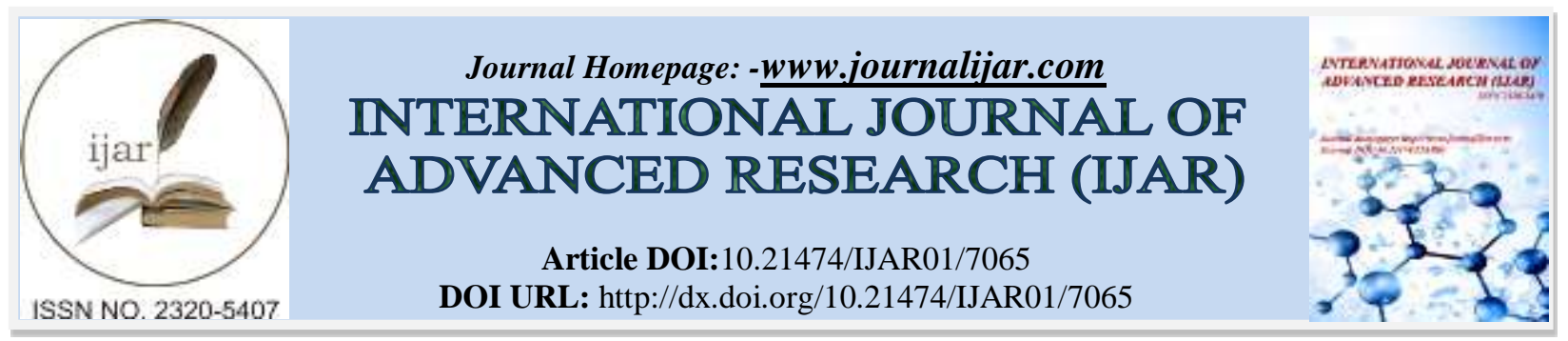

RESEARCH ARTICLE

\title{
CLINICAL VERSUS ULTRASONOGRAPHIC EXAMINATION IN CONGENITAL DYSPLASIA OF HIP JOINT.
}

\author{
Mustafa Elsagheir ${ }^{1}$, Rashid Darbauk ${ }^{1}$, Ala Wafa ${ }^{2}$ and Wisam Abozaid ${ }^{1}$. \\ 1. Orthopedic department Aljazeera Hospital, Misurata Libya. \\ 2. General Surgery department Aljazeera Hospital, Misurata Libya.
}

\section{Manuscript Info}

Manuscript History

Received: 08 March 2018

Final Accepted: 10 April 2018

Published: May 2018

Keywords:-

Congenital dysplasia of hip

Ultrasonographic examination of hip

joint ,Clinical examination of hip joint.

\section{Abstract}

Background: Congenital dysplasia of hip joint $(\mathrm{CDH})$ is one of the congenital anomalies in newborn that if not diagnosed and treated on time can lead to a severe instability. Although clinical examination is very useful way for screening, but in some patients a confirmatory diagnostic method such as Ultrasonography is needed. The aim of the present study is to compare the sensitivity and specificity of clinical examination and ultrasonography in early detection of $\mathrm{CDH}$.

Material and Methods: A total of 1000 newborn were examined by orthopedic surgeons as screening method. The newborns with risk factors or suspicious on clinical examinations were introduced to repeat clinical and ultrasonographic examination of hip joint. The results were collected and recorded by a check list and their sensitivity and specificity of clinical examination were calculated.

Results: There were about 980 newborns (1,960 hips) who were studied by two methods of clinical examination and ultrasonography (by Graf Method), the overall incidence of CDH was 33 per 1000. Only $13.5 \%$ of the disordered hips according to clinical examination were involved on ultrasonographic evaluation and the remaining $72 \%$ involved hips according to ultrasonography (Graf Type IIIb or Merze) were diagnosed Normal on clinical examination. Considering ultrasonography as gold standard method of evaluating $\mathrm{CDH}$, the sensitivity and specificity of clinical examination were calculated $29 \%$ and $93 \%$ respectively.

Conclusion: According to present study, ultrasonographic examination has high valuable in screening of $\mathrm{CDH}$ and clinical examination done by experienced orthopedic surgeon has an acceptable value in primary screening of $\mathrm{CDH}$ in developing countries for detecting healthy neonates, but if the newborn has a risk or is suspicious on clinical examination, it will be necessary to have assistance from ultrasonography by an experienced sonographer. 


\section{Introduction:-}

Congenital dysplasia of hip (CDH) is either complete dislocation or just sublaxation of femoral head and acetabular dysplasia (Herring, 2008). The word dysplasia consists of a disorder occurred during growth and development of hip in which, acetabulum, proximal of femur, and joint capsule are dynamically changing and making deformation.

The primary cause of instability in normal hip is laxity in joint capsule. Familial or inherited generalized laxity exists in high proportion of $\mathrm{CDH}$ patients, also hormonal and mechanical as the other risk factors.

Female to male ratio is 5-7 times. Other effective risk factors like breech presentation, first delivery, oligohydramnios, torticollis, cesarean section...

All neonates should be examined in the nursery by an experienced person, for example an orthopedist, a pediatrician, or an obstetrician, midwifery or an orthopedic doctor by doing Ortolani and Barlow tests. The suspicion of an abnormal hip will be increased if there is any risk factor in the neonate. Although their presence is helpful and shows a hip dislocation or sublaxation, their absence does not rule out the diagnosis of CDH and it needs a confirmatory tool like ultrasonography.

Also, clinical findings are different according to child age and the type of dislocation; diagnostic tool also varies in different ages. For example, ultrasonography is useful between 5-8months of age but not after 8-13 months.

The role of Ultrasonographic examination is not well defined because of its cost and dependency on the examiner. So the aim of the present study is comparison of sensitivity and specificity of clinical versus ultrasonographic examination in diagnosis of (Congenital) neonatal hip dysplasia.

\section{Materials and Methods:-}

A prospective study between January 2014- December 2016 about 1,000 child were examined by an orthopedic surgeons in *Aljazeera Hospital * at the end of $1^{\text {st }}$ and $6^{\text {th }}$ week and underwent ultrasonographic evaluation at the end of 6 week of birth. All the infants underwent clinical examination and ultrasonographic evaluation again were followed to 2 years of age.

During the study, about 20 neonates did not take part in follow-up (escaped) and were omitted from the study. The remaining 980 patients were evaluated by the two methods: clinical and ultrasonographic evaluation.

The inclusion criteria and exclusion criteria were taken according to risk factors and musculoskeletal disorders (Neural tube defect JCO Neonates) respectively.

After doing clinical examination and recoding the results, ultrasonographic examination was done by Sonoscope 7.5 $\mathrm{mHz}$ Linear transducer Sonalayer SSA-270. The ultrasonopgraphic evaluation were done by experienced sonographer and results were detremined according to Graf Classification. were not aware of the clinical examination results in this study. So the patients with normal developing hips (Graf type Ia and Ib) did not need follow-up, but hips with delay development (Graf IIa) were followed for 3 months and if no development occurred during this period were included in Graf IIb group. Neonates with Graf type IIb and the ones who were included in Graf type IIc, IId, III or IV on their first ultrasonographic evaluation were considered as CDH.

The clinical examination includes Ortolani and Barlow tests were accomplished by the authors.

\section{Results:-}

Total of 1000 patients underwent clinical examination of which 20 patients did not came up for follow-up. The remaining 980 patients (1,960 hips) were studied by two methods: clinical and ultrasonoraphic examination. 390 of the neonates (39\%) are male and 590 (61\%) are female In our study the distribution of (1,960 hips) were evaluated according to Graf Classification are as follows: 


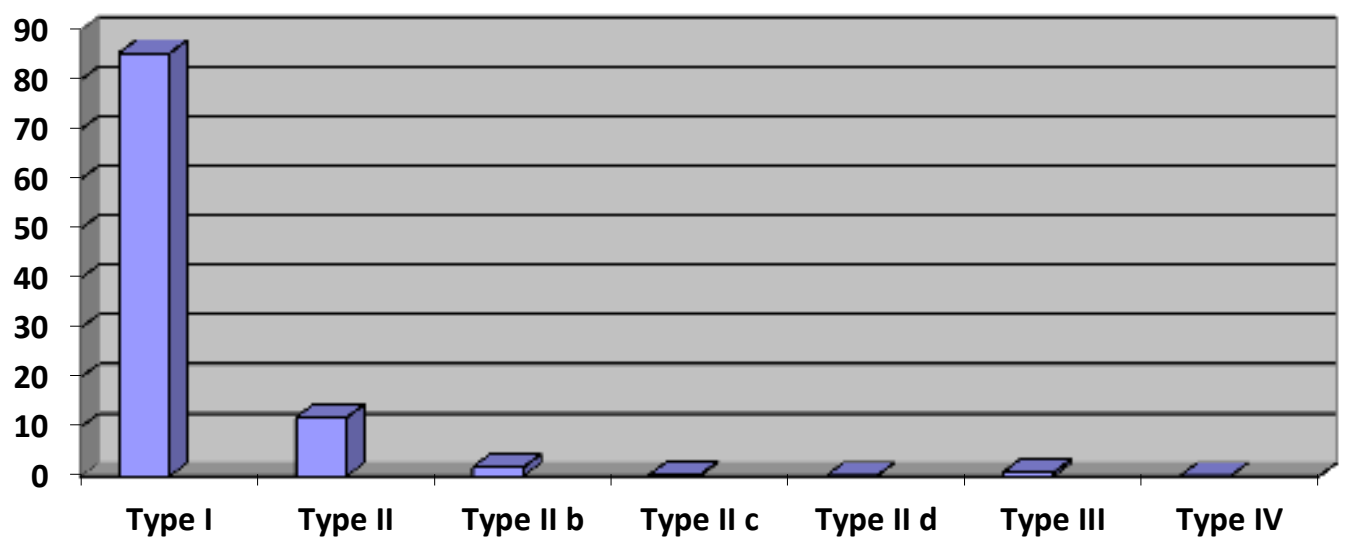

Figure 1:-Percentage Distribution according to Graf Classification

Type I hip - 85.3\%, Type IIa (physiologic immature) - 11.8\%, Type IIb (acetabular dysplasia) - 1.8\%, Type IIc (Critical zone) $-0.3 \%$, Type IId (sublaxation) $-0.07 \%$, Type III(mild dislocation) $-0.8 \%$, and Type IV (dislocated) $-0.04 \%$.

Clinical examination versus ultrasonographic in terms of number of hips defined as normal or pathologic shown on the table below.

Table 1:-Results of clinical and ultasonographic examination

\begin{tabular}{|c|c|c|c|}
\hline & Normal & Pathologic & $\begin{array}{c}\text { Percentage of diasesed } \\
\text { hip }\end{array}$ \\
\hline Clinical examination & 1787 & 173 & $8.8 \%$ \\
\hline $\begin{array}{c}\text { Ultrasonography } \\
\text { examination }\end{array}$ & 1886 & 74 & $3.8 \%$ \\
\hline $\begin{array}{c}\text { Final conclusion about } \\
\text { the hip joint condition }\end{array}$ & 1870 & 90 & $4.6 \%$ \\
\hline
\end{tabular}

The number of bilateral hip dislocation was $22 \%$ from total case And about $88 \%$ unilateral... about $73 \%$ of unilateral dislocation in the left hip Considering the ultrasonography as a gold standard method in evaluating $\mathrm{CDH}$, the sensitivity and specificity of clinical examination were determined as $30 \%$ and $90 \%$ respectively.

\section{Discussion:-}

The clinical examination of neonatal hip is a part of neonatal and infantile assessment but is not always enough in diagnosis of $\mathrm{CDH}$ alone. In some dysplasia, unstable, sublaxated or dislocated hips especially if examined by inexperienced person, diagnosis cannot be made or a normal hip may falsely be considered pathologic (false positive).

According to our study, ultrasonography is precise, sensitive and non-invasive method in diagnosis of CDH and abundance of joint cartilage in femoral head and acetabulum increases the sensitivity of the ultrasonography but if done in the first days after birth leads to false positive results due to joints capsular laxity. To prevent this, clinical and ultrasonographic examination was repeated in 6 weeks after birth and the results of final examinations were evaluated. Beside the experiences of the examiners and sonographer has an essential role in making differences. In our study, we took assistance from experienced orthopedic surgeons and sonographer.

The results of clinical hip examination and ultrasonographic reports were similar in $88 \%$ and different ultrasound results in $11 \%$ of neonates with normal hips according to clinical examination were reported. Also in $6 \%$ of cases with pathologic hip according to reports of clinical examination and ultrasonographic reports were mentioned normal results. The reason of this mismatch may as follows: The structure of hip joint in early birth so that the soft tissue and the capsular laxity around the hip joint and immature hip can naturally be existing in first few days to 
weeks and this immature laxity of hip, although, not so considered to make usual clinical tests positive, can be detected on ultrasonographic examination. Although accuracy and experience of the examiner in detecting cases of hip dislocation or subluxation is very important, we cannot imagine all the mismatched results due to the lack of the examiner experience. Besides the Barlow and Ortolani tests usually remain positive only during a few weeks (till 23 months) and finally hip will be fixed in a stable dislocated or reduced position. On the contrary, determining the quality of click or clunk sound is subjective perception and controversy on its accuracy is always existed by considering placing a vast range from stable and normal to having sever laxity and complete dislocated, the quality of specific click sound will be less important and any kind of click sound from hip is important, also, although orthopedic surgeons are the best ones who can perform neonatal hip examination, the routine examinations are not usually accomplished by them, of course it must be mentioned besides, that repeated clinical examinations can lead to loss of the hip vacuum stability and decrease of the hip stability.

Ultrasonography of hip needs enough and great experience in detecting instability and dislocation and the more ultrasonographies carried out by the specialist, the less the errors will take place so that Harcke recommends: If there is not enough experience on hip ultrasonography, its report should be prevented. Beside the specialist experience, also the neonate's restlessness during examination can lead to make mistakes in diagnosis both in clinical and ultrasonographic examination. Finally, it must be confirmed that some of the hips having subluxation or dislocation on ultrasonography may have negative Barlow or Ortolani test, so loss of positive tests is not the reason for the lack of dislocation on the ultrasonography. The number of palpable click sounds decreases with the neonatal growth and this is not always leading to hip stability but can be due to stabilizing in a dysplastic position of the hip. A positive ortolani test often remains positive only for a few days. The test may become negative due to hip stabilization or it may become stabilized in a deblocking position and the reduction will be difficult, so during this period to appearance of femoral head on the radiographs, ultrasonography is the best diagnostic tool.

Although primarily it was thought that if clinical examination in all newborns can solve the problem of CDH as a dilemma for patients and occasionally physicians, now it is shown in most center which are carried out, the neonatal clinical examination will not detect some cases of $\mathrm{CDH}$ alone or may lead to unnecessary treatments.

Although some false-positive cases may be existed (if left untreated, the $\mathrm{CDH}$ will not develop later), considering the prevalence of false-negative cases (normally reported on the screening but develop dislocation later) and the incidence of $\mathrm{CDH}$ in the study about 29 per 1000 newborns it can be recommended that the best, fastest, least expensive, and most useful method of $\mathrm{CDH}$ screening in developing countries is clinical examination by an experienced person. If possible, all the neonates or suspicious and with risk factors neonates should be reexamined by an orthopedic surgeon after primary examination by the obstetrician in the delivery ward or the midwifery or pediatrician.

Although now today for detecting $\mathrm{CDH}$ a clinical sound or click or absence of it is not confirmatory, then ultrasonography should no longer be regarded as a screening method but should be used as an investigative and confirmatory tools in neonates to clarify and distinguish the diagnosis. Ideal screening test must be simple, reliable with high levels of sensitivity and specificity, and providing cost-effective results. Since these criteria are not fulfilled in cases of $\mathrm{CDH}$, the screening term may be converted to surveillance as a more appropriate suggestive alternative word. On the basis of our data and taking into consideration all possible conditions related to $\mathrm{CDH}$, we conclude that, along with clinical examination to detect $\mathrm{CDH}$, ultrasonography screening should be added to highrisk neonatal screening program.

\section{Conclusion:-}

According to present study, ultrasonographic examination has high valuable in screening of CDH and clinical examination done by experienced orthopedic surgeon has an acceptable value in primary screening of CDH in developing countries for detecting healthy neonates, but if the newborn has a risk or is suspicious on clinical examination, it will be necessary to have assistance from ultrasonography by an experienced sonographer.

\section{Advice:-}

1. Ultrasonography examination and clinical examination should be done together and you cannot depend any one of this alone

2. Ultrasonography is gold standard method of evaluating $\mathrm{CDH}$

3. Ultrasonography is preferred after age of 4 wks due to false positive result in first wks 
4. Early diagnosis of C.D.H leading to prevent a lot of

5. Catastrophic complication and disability

6. Expert orthopedic and sonographer doctor should manage and dealing with C.D.H cases

7. Early detection of C.D.H cases leading to make the society away from surgical

\section{References:-}

1. Herring JA. $4^{\text {th }}$ ed. Philadelphia: Saunders Elsevier, 2008. Developmental Dysplasia of the hip. Tachdjian's pediatric orthopedics from the Texas Scottish Rite hospital for children; pp.637-8

2. Kokavec M, Bialik V. Developmental dysplasia of the hip. Prevention and real incidence. Bratisl Lek Listy. 2007;108:251-4 (Pubmed)

3. Ortolani M. Congenital hip dysplasia in the light of early and very early diagnosis. Clin Orthop relat Res. 1976:6-10 (PubMed)

4. Herring JA. $4^{\text {th }}$ ed. Philadelphia: Saunders Elsevier; 2008. Developmental Dysplasia of the Hip: Tachjidian's pediatric Orthopadeics From The Texas Scottish Rite Hospital for Children; pp. 639-41

5. Patron RW, Hinduja $\mathrm{K}$, Thomas $\mathrm{CD}$. The significance of at-risk factors in ultrasound surveillance of developmental dysplasia of the hip. A ten-year prospective study. J Bone Joint Surg Br. 2005;87B:1264-6.

6. Mahdi Alsaleem, Kallol K. Set and Lina Saadeh, Developmental Dysplasia of Hip, clinical pediatrics, 54, 10, (921), (2015).

7. I. H.Feeley, C. J .Green, F. E. Rowan and D. P. Moore, International Variance in the treatment of developmental dysplasia of the hip, Journal of Children's Orthopedics, 8, 5, (381), (2014).

8. James Tomlinson,Dominic O'Dowd and James Alfred Fernandes, Managing Developmental Dysplasia of the Hip, The Indian Journal of Pediatric , 83, 11, (1275), (2016).

9. Robin W. Paton, Screening in Developmental Dysplasia of the Hip (DDH), The Surgeon, 15, 5, (290), (2017).

10. Storer S. K., Skaggs D. L. Developmental dysplasia of the hip. Am Fam Physician. 2006;74(8):13101316.[PubMed]

11. Dezateux C., Rosendahl K. Developmental dysplasia of the hip. Lancet. 2007;369(9572):1541-1552. [PubMed]

12. Sewell M. D., Rosendahl K., Eastwood D. M. Developmental dysplasia of the hip. BMJ. 2009 Nov 24;339:b4454. [PubMed]

13. Klisic P. J. Congenital dislocation of the hip a misleading term: abrief report. JBone Joint Surg Br.1989;71:136.[PubMed]

14. Sankar W. N., Weiss J., Skaggs D. L. Orthopedic conditions in the newborn. J Am Acad Orthop Surg. 2009;17(2):112-122.[PubMed]

15. McEvoy A., Paton R. W. Ultrasound compared with radiographic assessment in developmental dysplasia of the hip. J R Coll Surg Edinb. 1997;42:254-255. [PubMed] 